\title{
Challenges in Formulation and Implementation of Hepatitis B Elimination Programs
}

\author{
${\text { Zaigham } \text { Abbas }^{1} \text {, Minaam Abbas }}^{2}$ \\ 1. Gastroenterology and Hepatology, Dr. Ziauddin University Hospital, Karachi, PAK 2. Medicine, School of Clinical \\ Medicine, University of Cambridge, Cambridge, GBR
}

Corresponding author: Zaigham Abbas, drzabbas@gmail.com

\begin{abstract}
Nearly 257 million individuals have contracted hepatitis B infection around the world. However, only $10 \%$ of them know about their illness. Mother to child transmission, nosocomial spread, and sexual transmission are the major etiological factors. Finding the missing millions is a global issue. Hepatitis B care is more difficult compared to hepatitis $C$ as not all patients require treatment and the selection of patients is not straightforward. To eliminate hepatitis B infection, the program should screen pregnant women and start antiviral therapy from the 28th week of pregnancy if hepatitis B virus (HBV) DNA $\geqslant 200,000 \mathrm{IU} / \mathrm{mL}$ or hepatitis B e-antigen (HBeAg) reactive. Prevention of perinatal infection, birth dose and neonatal vaccination, post-vaccination monitoring of high-risk groups, catch-up vaccination, and registration of the carriers should be an integral part of the program. Continuum of care is important when planning the elimination program from addressing the risk factors, testing, and referral for treatment. The program should integrate test and treat hepatitis services with existing local health care services. There is a need to create the right environment, raise awareness, remove stigma, and increase screening of those at risk and manage those who require treatment. A national policy should be prepared for capacity building, fund allocation, and implementation strategies. Micro-elimination strategies should boost national elimination effects. Guidelines to diagnose and treat patients with hepatitis B should be simplified. Surveillance should be done to monitor progress, and determine the impact of the elimination program on incidence and mortality, and services.
\end{abstract}

Review began 03/24/2021 Review ended 04/17/2021 Published 04/24/2021

() Copyright 2021

Abbas et al. This is an open access article distributed under the terms of the Creative Commons Attribution License CC-BY 4.0., which permits unrestricted use, distribution, and reproduction in any medium, provided the original author and source are credited.

Categories: Gastroenterology, Public Health

Keywords: who, elimination programs, vaccination, mother to child transmission, nosocomial, hepatitis b(hbv)

\section{Introduction And Background}

Worldwide, about 257 million persons have been exposed to hepatitis B (2015 estimates). Only 10\% of hepatitis B patients know about their disease [1]. If left untreated, about $25 \%$ of infected individuals will progress to cirrhosis or hepatocellular carcinoma (HCC) [1-3]. Every year, 900,000 patients die of hepatitis B and its complications. Around $58 \%$ of children globally do not have access to the birth dose of the hepatitis B vaccine. Unfortunately, as of 2017 , only $28 \%$ of countries have interventions to support hepatitis B virus (HBV) elimination plans, citing cost as the primary impediment [1]. Coronavirus disease 2019 (COVID-19) has badly affected all the services related to hepatitis elimination [4]. Consequently, it seems difficult to achieve the WHO target of elimination of viral hepatitis as a major public health threat.

\section{Review}

\section{WHO goals for viral hepatitis elimination}

In May 2016, the WHO Global Health assembly released its goals to eliminate viral hepatitis as a major public health threat by 2030 [5]. This included some important targets: $90 \%$ of infants should have a birth dose vaccination for hepatitis B, HBsAg (hepatitis B surface antigen) prevalence should be below $0.1 \%$ in children 5 years of age, $100 \%$ of blood donations should be screened for viral hepatitis (both B and C), and $90 \%$ of injections should be administered with safe syringes. For testing, $90 \%$ of individuals with viral hepatitis should be aware of their infection, and $80 \%$ of those infected should be treated. This would result in a $90 \%$ reduction in the incidence of new infections and a $65 \%$ reduction in mortality. However, according to the forecast HBsAg prevalence is not going to change assuming the status quo, many countries will only to able to achieve some of the set goals later than the year 2050. Only three countries are expected to achieve a $90 \%$ reduction in HBV incidence, none will achieve a $65 \%$ reduction in mortality relative to 2015 , and no country will achieve all the current HBV elimination targets [6].

\section{Formulating an elimination program}

When formulating a hepatitis B elimination program, we should know about the disease burden to deal with. What are the risk factors? What are the targets? Numbers needed to screen and treat? The capacity and cost issues, policy, guidelines, financing, and implementation strategies should be kept in mind. The program should focus on prevention, which means safe blood and infection control, vaccinations, harm reduction 


\section{Cureus}

measures for individuals participating in high-risk activities, and prevention of mother-to-child transmission. The facilities for testing and initiation of therapy among those already infected should be expanded. Diagnosis and treatment are really important when striving toward elimination. Barriers to the effective implementation of hepatitis control programs should be preempted and properly addressed (Table 1) [7-9].

Lack of diagnostic and treatment capacity

Scarce and expensive medicines

Lack of dissemination of information via media involvement

Poor routine immunization

No birth dose vaccine

Lack of routine maternal screening

Unawareness about treating hepatitis B during pregnancy

Poor compliance to follow the schedule of $0,1 \& 6$ months for hepatitis B vaccine

A disconnect between duty bearers and right holders

Lack of coordination among public/private and private/private players

Low political priority

Lack of funding from government and donor agencies

The large increase in hepatitis due to the persistence of risk factors and new case findings

An outdated health care system

Lack of awareness/education both on the supply side (health care providers) and demand-side (community)

Lack of trained specialists and health workers

Quackery

COVID-19 pandemic

TABLE 1: Barriers to effective implementation of hepatitis control programs in the developing countries

\section{Risk factors for HBV infections}

Worldwide mother-to-child transmission is a prime risk factor [10]. Sexual transmission also exists. The nosocomial spread is still a major issue in developing and poor countries. Multiple therapeutic injections, unsafe blood transfusions, reuse or sharing of needles and syringes, surgical and dental procedures, and needle-stick injuries in healthcare workers, all are contributing factors [11]. All these healthcare-related issues are modifiable and policies and procedures are needed to be developed and enforced to ensure that the transmission of HBV is halted.

\section{Find the missing millions}

Finding the missing millions is a global issue. The infection is hugely under-diagnosed and under-treated even in the most developed countries [12]. About $80 \%$ of carriers of hepatitis B and C are undiagnosed. What are the reasons for missing millions of infected people with hepatitis $\mathrm{B}$ ? We are not doing the mass screening. It is of low political priority. There is a lack of sufficient knowledge about hepatitis B and C and risk factors. Those at risk do not demand to screen as they are asymptomatic and feeling well or ascribe their symptoms to other things, many of these hail from underprivileged communities, and stigma and discrimination after diagnosis of hepatitis B exist.

Family doctors take little action to motivate their patients to get tested for hepatitis B and C [13]. They may be too busy or less motivated for case-finding. There may be a lack of knowledge of symptoms until endstage liver disease with complications occurs. Due to a lack of understanding of abnormal liver function 
tests (LFTs), they dismiss subtle changes. A disparity exists between urban and rural populations in terms of physician's approach, access to diagnostics, and treatment.

\section{Case finding and therapy is cost-effective}

It has been shown that case-finding and therapy for chronic viral hepatitis in primary care are potentially difficult with community-based interventions even in developed countries [13]. However, screening for viral hepatitis in primary care by incentivized practitioners appears cost-effective and helps to identify a cohort of motivated individuals who will engage with treatment. A study done on HBsAg prevalence in the Gambia based on the Markov state transition model confirms that community-based screening and treatment for chronic hepatitis $B(\mathrm{CHB})$ is cost-effective in regions with an intermediate and high prevalence of hepatitis B [14]. A population-based, cluster-randomized, controlled trial between 1985 and 1990 from China confirms that the cumulative incidence probability of HCC and cumulative mortality probability of liver diseases in the vaccination group is much less compared to the control group [15]. Seroprevalence of hepatitis B virus in Taiwan 30 years after the commencement of the national vaccination program has decreased to less than $1 \%$ in university students which used to be about $10 \%$ [16]. The strategy to completely eradicate hepatitis B infection in Taiwan included general preventive hygiene caution measures and an effective anti-viral therapy if indicated, screening of pregnant women, perinatal infection prevention, neonatal vaccination, post-vaccination monitoring of high-risk groups, and registration of carriers and management adequately [17].

A systematic review addressed hepatitis B screening with economic evaluations in low- and middle-income countries (LMIC). Nine studies fulfilled the eligibility criteria. Screening with 'catch-up' vaccination for younger adults yielded benefits above costs, and screening linked with treatment showed cost-effectiveness that might be affordable for some LMICs [18].

\section{WHO 2020 recommendations: pregnant women}

Recently, on World Hepatitis Day, WHO released its guidelines to prevent mother-to-child transmission of hepatitis B [19]. Pregnant women testing positive for HBV infection with an HBV DNA $200,000 \mathrm{IU} / \mathrm{mL}$ should receive tenofovir prophylaxis from the 28th week of pregnancy until at least birth, to prevent motherto-child transmission of HBV. This is in addition to three-dose hepatitis B vaccination in all infants, including a timely birth dose. If HBV DNA testing is not available, hepatitis B e-antigen (HBeAg) testing can be used as an alternative to HBV DNA testing to determine eligibility for tenofovir prophylaxis. The systematic reviews of antiviral therapy during pregnancy to prevent mother-to-child transmission (MTCT) favour treatment $[19,20]$

WHO suggests an Incremental approach to the prevention of HBV infection at birth and in the first years of life it includes maternal antiviral prophylaxis as mentioned above, HBsAg testing linkage to care and followup of infants, when available hepatitis B immunoglobulin for infants born to HBsAg and HBeAg positive mothers, and at least three doses of hepatitis B vaccine, including a birth dose within 24 hours.

\section{Treatment of hepatitis B in elimination programs}

HBV care is more difficult compared to HCV (Table 2). 


\section{Cureus}

\begin{tabular}{|l|l|l|}
\hline & Hepatitis C & Hepatitis B \\
\hline Vaccination & No vaccine & Birth dose vaccine a real challenge \\
\hline Mother to child transmission & Not a major issue & A real threat \\
Diagnostic tests & Accessible and cheaper & Cost barriers \\
Treatment & Curable & Treatable \\
Eligible for treatment & All PCR positive patients & Selected patients \\
Drugs to treat & Cheap generics available & Issue in some countries \\
New drugs development & Occurred at a rapid pace & Slow \\
Awareness, advocacy & Increased after availability of directly acting antivirals (DAAs) & Community movements lacking \\
Monitoring and follow-up & Easy & Difficult with many steps
\end{tabular}

\section{TABLE 2: Differences between hepatitis $C$ and $B$ elimination}

Continuum of care is important when planning the elimination program from testing to referral and treatment [7]. Hepatitis control programs should focus on training and educating staff to follow algorithms to deliver initial care. As not all patients require treatment for hepatitis B, the selection of patients is not straightforward in the case of hepatitis B. Patients who fulfil the eligibility criteria should be selected for treatment. Proper training of primary care physicians may minimize the requirements for specialist care and the number of visits as proposed in Figure 1.

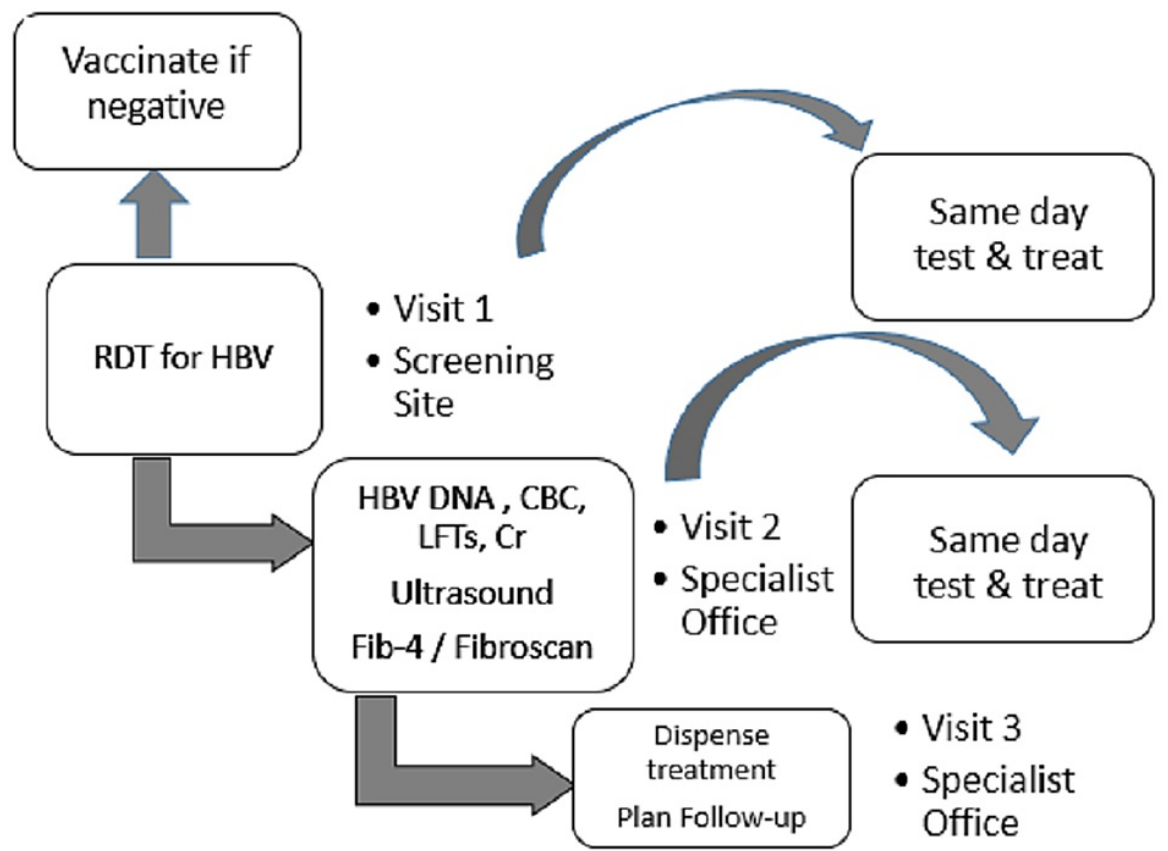

FIGURE 1: How to decentralize and simplify the service delivery in the hepatitis B elimination program

RDT, rapid diagnostic tests; HBV, hepatitis B virus; DNA, deoxyribonucleic acid; CBC, complete blood counts; LFTs, liver function tests; Cr, creatinine; Fib-4, fibrosis-4

The program should integrate services for managing viral hepatitis with existing health care services and 
work on improving safety, e.g., screening of blood products, provision of clean needles, and infection control in healthcare facilities. With the availability of generic drugs to treat hepatitis $\mathrm{B}$, the cost of treatment has decreased drastically. Treatment with nucleoside analogues is safe and effective.

\section{Micro-elimination strategies of hepatitis B}

Micro-elimination strategies target individual population segments for which treatment and prevention interventions can be delivered quicker and more efficiently. These programs may address districts with high prevalence, identify pockets of high prevalence within districts, take measures to prevent transmission or address risk factors, and identify target groups to treat infection and prevent transmission. For example, these programs may focus on antenatal screening, infant vaccination, catch-up vaccination, vaccination of persons who inject drugs, prisoners, decompensated cirrhosis, veterans, or patients with haemophilia and homosexuals. It has been shown by creating a lifetime Markov model that strategies to vaccinate, prevent or treat CHB in high-risk populations result in a significant reduction in cirrhosis, decompensation, liver cancer, and chronic hepatitis death with the intervention compared to no intervention [21].

Micro-elimination strategies are tailored with realistic and well-defined targets and goals. These are pragmatic, time to achievement is shorter and cost can be predicted. Micro-elimination projects may generate a template in a small geographically defined population which may then be used to model services for larger intervention programs. Successful micro-elimination efforts encourage further public health strategies. Micro-elimination of hepatitis B, appear cost-effective and will have a positive impact on longterm outcomes with screen and treat or vaccinate strategy compared with no intervention.

\section{Lessons from the successful Egyptian program}

Egypt is running a very successful hepatitis control program and is achieving the WHO set targets [22,23]. All hospitals and rural health centres were involved. A screening centre was set up in every village. Mobile units were made available. A huge media campaign was started through TV, radio, social media, paper media, and SMS (short message service). A successful awareness campaign was run and society pressure generated. A person could walk into any screening centre. Rapid diagnostic tests were made available instead of ELISA (enzyme-linked immunosorbent assay). Seropositive patients were immediately referred to an evaluation centre. Free investigations and treatment were available. Call centres were set up to prevent dropouts and contact no-shows. The management guidelines were made simple. The focus was to decrease the number of visits. There was a continuous political will and support. The government allocated sufficient resources and funds to initiate and maintain the program. An empowered central decision-making body was set up.

\section{How to scale up elimination and meet the targets}

Public Health is a great issue in low-income countries where the cost of diagnostic tests is high keeping in mind that due to the unavailability of government facilities $70 \%$ of the population visits the private sector for health issues. Around 60-70\% of expenditure is out of pocket. It is impossible to control hepatitis without the availability of free tests and treatment facilities. Guidelines to diagnose and treat patients with hepatitis B are to be made simplified, infrastructure to test and treat should be expanded and targets should be set up to test and treat in local health care centres. Sufficient funds should be allocated ad Innovative financing strategies should be devised to raise the money $[7,11,24,25]$.

\section{Validation of elimination efforts}

Surveillance should be done to monitor progress, and determine the impact of the elimination program on incidence and mortality, and services. Monitoring may be done by conducting two surveys of national or high-risk groups a minimum of one year apart and estimate incidence between the two by age group [26]. There is a need to establish a national registry for decompensated cirrhosis and HCC linked to patient and death registries. Modelling may be used to estimate incidence when doing surveys is not possible.

\section{Conclusions}

There is a high prevalence of HBV in many regions of the world. The disease burden will not decrease through 2030 with the continuation of the base strategy. Control of hepatitis B is feasible in the next 10 years or so through an aggressive national strategy that requires political will, strategic plans with timelines, multiple stakeholders' involvement, strong infrastructure, public awareness, and funding (domestic and international resources). For harm reduction, a broad education campaign, control of nosocomial infections, treatment of pregnant ladies with high viral load, and vaccination including birth dose vaccination is warranted. A scale-up in screening is required to keep pace with the increase in treatment. A significant increase in the number of individuals being treated will mitigate the disease burden. Access to therapy is important. The availability of generics would make this endeavour more effective.

\section{Additional Information \\ Disclosures}


Conflicts of interest: In compliance with the ICMJE uniform disclosure form, all authors declare the following: Payment/services info: All authors have declared that no financial support was received from any organization for the submitted work. Financial relationships: All authors have declared that they have no financial relationships at present or within the previous three years with any organizations that might have an interest in the submitted work. Other relationships: All authors have declared that there are no other relationships or activities that could appear to have influenced the submitted work.

\section{References}

1. Hepatitis B. Accessed: March 20, 2021: https://www.who.int/news-room/fact-sheets/detail/hepatitis-b.

2. Fattovich G, Bortolotti F, Donato F: Natural history of chronic hepatitis B: special emphasis on disease progression and prognostic factors. J Hepatol. 2008, 48:335-52. 10.1016/.j.jhep.2007.11.011

3. McMahon BJ: The natural history of chronic hepatitis B virus infection . Hepatology. 2009, 49:S45-55. 10.1002/hep.22898

4. Wingrove C, Ferrier L, James C, Wang S: The impact of COVID-19 on hepatitis elimination . Lancet Gastroenterol Hepatol. 2020, 5:792-4. 10.1016/S2468-1253(20)30238-7

5. World Health Organization: Global Health Sector Strategy on Viral Hepatitis 2016-2021 . World Health Organization, Geneva; 2021.

6. Polaris Observatory. (2021). Accessed: March 20, 2021: https://cdafound.org/dashboard/polaris/maps_prev.html.

7. Cooke GS, Andrieux-Meyer I, Applegate TL, et al.: Accelerating the elimination of viral hepatitis: a Lancet Gastroenterology \& Hepatology commission. Lancet Gastroenterol Hepatol. 2019, 4:135-84. 10.1016/S24681253(18)30270-X

8. Thomas DL: Global elimination of chronic hepatitis. N Engl J Med. 2019, 380:2041-50. 10.1056/NEJMra1810477

9. Tseng TC, Kao JH: Elimination of hepatitis B: is it a mission possible? . BMC Med. 2017, 15:53. 10.1186/s12916-017-0820-X

10. Yi P, Chen R, Huang Y, Zhou RR, Fan XG: Management of mother-to-child transmission of hepatitis B virus: Propositions and challenges. J Clin Virol. 2016, 77:32-9. 10.1016/j.jcv.2016.02.003

11. Waheed Y, Siddiq M, Jamil Z, Najmi MH: Hepatitis elimination by 2030: progress and challenges . World J Gastroenterol. 2018, 24:4959-61. 10.3748/wjg.v24.i44.4959

12. Rajbhandari R, Chung RT: Screening for hepatitis B virus infection: a public health imperative . Ann Intern Med. 2014, 161:76-7. 10.7326/M14-1153

13. Flanagan S, Kunkel J, Appleby V, et al.: Case finding and therapy for chronic viral hepatitis in primary care (HepFREE): a cluster-randomised controlled trial. Lancet Gastroenterol Hepatol. 2019, 4:32-44. 10.1016/S2468-1253(18)30318-2

14. Nayagam S, Conteh L, Sicuri E, et al.: Cost-effectiveness of community-based screening and treatment for chronic hepatitis B in The Gambia: an economic modelling analysis. Lancet Glob Health. 2016, 4:568-78. 10.1016/S2214-109X(16)30101-2

15. Qu C, Chen T, Fan C, et al.: Efficacy of neonatal HBV vaccination on liver cancer and other liver diseases over 30-year follow-up of the Qidong hepatitis B intervention study: a cluster randomized controlled trial. PLoS Med. 2014, $11:$ e1001774. 10.1371/journal.pmed.1001774

16. Hu YC, Yeh CC, Chen RY, et al.: Seroprevalence of hepatitis B virus in Taiwan 30 years after the commencement of the national vaccination program. PeerJ. 2018, 6:e4297. 10.7717/peerj.4297

17. Ni YH, Chang MH, Jan CF, Hsu HY, Chen HL, Wu JF, Chen DS: Continuing decrease in hepatitis B virus infection 30 years after initiation of infant vaccination program in Taiwan. Clin Gastroenterol Hepatol. 2016, 14:1324-30. 10.1016/j.cgh.2016.04.030

18. Wright CM, Boudarène L, Ha NT, Wu O, Hawkins N: A systematic review of hepatitis B screening economic evaluations in low- and middle-income countries. BMC Public Health. 2018, 18:373. 10.1186/s12889-0185261-8

19. Prevention of mother-to-child transmission of hepatitis B virus: guidelines on antiviral prophylaxis in pregnancy. (2021). Accessed: March 20, 2021: https://www.who.int/publications-detail-redirect/978-92-4000270-8.

20. Hyun MH, Lee YS, Kim JH, Je JH, Yoo YJ, Yeon JE, Byun KS: Systematic review with meta-analysis: the efficacy and safety of tenofovir to prevent mother-to-child transmission of hepatitis B virus. Aliment Pharmacol Ther. 2017, 45:1493-505. 10.1111/apt.14068

21. Chahal HS, Peters MG, Harris AM, McCabe D, Volberding P, Kahn JG: Cost-effectiveness of hepatitis B virus infection screening and treatment or vaccination in 6 high-risk populations in the United States. Open Forum Infect Dis. 2019, 6:ofy353. 10.1093/ofid/ofy353

22. Shiha G, Metwally AM, Soliman R, Elbasiony M, Mikhail NNH, Easterbrook P: An educate, test, and treat programme towards elimination of hepatitis $\mathrm{C}$ infection in Egypt: a community-based demonstration project. Lancet Gastroenterol Hepatol. 2018, 3:778-89. 10.1016/S2468-1253(18)30139-0

23. Schröeder SE, Pedrana A, Scott N, et al.: Innovative strategies for the elimination of viral hepatitis at a national level: a country case series. Liver Int. 2019, 39:1818-36. 10.1111/liv.14222

24. Martin NK, Vickerman P, Hickman M: How to eliminate HCV infection by antiviral treatment . J Hepatol. 2017, 67:5-6. 10.1016/j.jhep.2017.03.008

25. Hatzakis A, Lazarus JV, Cholongitas E, et al.: Securing sustainable funding for viral hepatitis elimination plans. Liver Int. 2020, 40:260-7. 10.1111/liv.14282

26. Polaris Observatory Collaborators: The case for simplifying and using absolute targets for viral hepatitis elimination goals. J Viral Hepat. 2021, 28:12-9. 10.1111/jvh.13412 